\title{
Determination of Amphetamines in Hair by GC/MS after Small-volume Liquid Extraction and Microwave Derivatization
}

\author{
Pinjia Meng, ${ }^{\dagger}$ Dan Zhu, Hongyuan He, Yanyan Wang, Fei Guo, and Liang Zhang \\ Department of Forensic Science, Chinese People's Public Security University, Beijing 100038, China
}

\begin{abstract}
We report here on the results of a procedure for the determination of amphetamine drugs in hair. The procedure is simple and sensitive. The results from the procedure using small-volume extraction matches perfectly with those either from using the derivatization method or selected ion monitoring (SIM) detection. We validated our method using four different amine drugs, including amphetamine, methamphetamine, methylenedioxy-amphetamine and methylenedioxymethamphetamine. The detection limit for these drugs is about $50 \pm 7.5 \mathrm{pg} / \mathrm{mg}$ in hair and the intra-day and inter-day reproducibility are within $15 \%$ at most drug concentrations. Moreover, we also showed the utility of the procedure in analyses of authentic hair samples taken from amphetamine abusers, and demonstrated that the method meets the requirement for the analysis of a trace amounts of amphetamines in human hair.
\end{abstract}

(Received July 7, 2008; Accepted December 24, 2008; Published September 10, 2009)

\section{Intruduction}

Hair has been recognized as an important biological specimen, along with urine and blood, for detecting drug abuses. It has several advantages, such as a long surveillance time window, easy sample collection, availability of repetitive sampling, and an excellent correlation between the "degree of addiction" and the actual drug consumption, over other types of specimen. ${ }^{1,2}$ Amphetamine (AM) and its derivatives are powerful stimulants of a central nervous system. The abuse of amphetamine, methamphetamine (MAM) and their methylenedioxy-derivatives, such as methylenedioxy-amphetamine (MDA), methylenedioxymethamphetamine (MDMA) and methylenedioxyethylamphetamine (MDE) has increased enormously during the last few years, causing serious health and social problems. In response to a growing demand for reliable detection, a number of methods have been developed to provide reliable results for the identification and quantification of drugs and related compounds in biological matrices. ${ }^{3-6}$ However, detecting trace amounts of these substances in biological matrices remains to be a challenge for analytical chemists.

Analyses of drugs in hair involve a number of steps, any one of which is critical for the sensitivity of drug detection. First, hair is processed under an alkali, acidic condition, ${ }^{6-8}$ or with an enzymatic treatment. ${ }^{2}$ This step is followed by either liquid-phase extraction (LPE), ${ }^{6,79}$ solid-phase microextraction (SPME), ${ }^{10-13}$ or supercritical fluid extraction (SFE). ${ }^{14}$ Second, concentrating and derivatizing the analytes, in order to increase the detection sensitivity, are performed. ${ }^{9,10}$ Lastly, the analytes are detected using gas chromatography/mass spectrometry (GC/MS), ${ }^{15,16}$ gas chromatography/mass spectrometry and mass spectrometry (GC/MS/MS), ${ }^{17}$ high-performance liquid chromatography (HPLC), ${ }^{6,9,18}$ and capillary electrophoresis

† To whom correspondence should be addressed.

E-mail: mengpinjia@163.com
(CE). ${ }^{4,7}$ These methods require certain instrumentation setups, such as SPME or CE or HPLC, and are therefore limited for their wide applications, especially in routine case or field work.

The aim of this study was to develop a sensitive, low-cost and simple procedure to analyze trace amounts of amphetamines in human hair. We applied simply low-cost small-volume liquid extraction, followed by applying rapid and complete derivatization under microwave heating, ${ }^{19}$ and by using GC/MS for detection using selected ion monitoring (SIM) mode. With the perfect compatibility of small-volume liquid extraction and derivatization, both of which were conducted in the same solvent, as well as the combination of derivatization and SIM detection. This method was simple and of sufficiently high sensitivity with limits of detection (LOD) suitable for the determination of drugs below the cut-off usually adopted for hair analysis $(0.1 \mathrm{ng} / \mathrm{mg}){ }^{7}$

In our procedure, we used a small volume $(50-100 \mu \mathrm{L})$, compared to a 10 -fold volume in the reported procedure, of an organic solvent; ${ }^{4,5,9}$ We used microwave heating to shorten the derivatizing time to within $3 \mathrm{~min}$, which is not applied widely, as reflected from the literature. Furthermore, in our procedure, there is neither a $\mathrm{pH}$ adjusting step that was used in acid extraction, ${ }^{5,8}$ nor phase-separating and solvent-evaporating steps, which were used in most hair-analysis procedures. Those steps are not only time-consuming, but also result in sample losses., ${ }^{4,5,9}$ Moreover, we demonstrated the utility of our procedure for analyzing hair samples from amphetamine abusers using small amounts of hair samples. The procedure is sensitive, and can be used to analyze trace amounts of amphetamines in human hair.

\section{Experimental}

Reagents and chemicals

$N$-Methyl-bis-trifluoroacetamide (MBTFA) was purchased from MACHEREY-NAGEL (Germany). 2-Methyl-benzylamine and heneicosane $\left(\mathrm{C}_{21} \mathrm{H}_{44}\right)$ (internal standards) were purchased 
Table 1 Qualitative and quantitative selective ions in GC/MS detection

\begin{tabular}{cccccc}
\hline & AM-TFA & MAM-TFA & MDA-TFA & MDMA-TFA & 2-Methyl-benzylamine-TFA (IS) \\
\hline Retention time/min & 6.17 & 7.61 & 10.34 & 11.67 & 7.51 \\
SIM $(m / z)$ Qualitative & $91,118,140$ & $110,118,154$ & $77,135,162$ & $135,154,162$ & 91,118 \\
Quantitative & 118 & 154 & 135 & 162 & 118 \\
\hline
\end{tabular}

from ACROS ORGANICS (Belgium). AM, MAM, MDA, MDMA were all purchased from the Evidence Identification Center of China (Beijing, China). All reagents used in this work were of analytical grade.

\section{Apparatus}

GC/MS analysis was carried out on a GC-MS hyphenated instrument (Agilent Technologies). The ionizing voltage was set at $70 \mathrm{eV}$, and the scan range was from 40 to $450 \mathrm{~m} / \mathrm{z}$. A $30-\mathrm{m}$ column with an internal diameter of $0.25 \mathrm{~mm}$ containing cross-linked and surface-bonded dimethyl-polysiloxane (DB-5) was used. The film thickness was $0.25 \mu \mathrm{m}$ (Agilent). For gas chromatographic analyses, the temperature for both the injector and the detector were $250^{\circ} \mathrm{C}$. The oven temperature was initially set at $100^{\circ} \mathrm{C}$, holding steady for $1 \mathrm{~min}$, followed by a ramp at a rate of $10^{\circ} \mathrm{C} / \mathrm{min}$ to $210^{\circ} \mathrm{C}$, then holding for $3 \mathrm{~min}$. The solvent delay for a mass spectrometer was $3 \mathrm{~min}$. Helium was used as a carrier gas at a flow rate of $1.0 \mathrm{~mL} / \mathrm{min}$. Splitless injection was used.

\section{SIM application}

Three selected ions with higher abundance and specificity in mass spectroscopy of derivatized standards were used for qualitative monitoring of the drugs. Among them, one ion for each drug was used for quantitative analyses. Table 1 lists the selective ions.

\section{Hair sample preparation}

Blank hair samples were obtained from a fifteen-year old male juvenile, who was free of illicit drug abuse. The hair samples were washed with a detergent to remove any lipids from the surface, and were rinsed with tap water, followed by distilled water to remove the detergent. The hair samples were then dried at room temperature and cut for the next steps. The blank hair samples were spiked with standard drugs with concentrations from 0.4 to $5 \mathrm{ng} / \mathrm{mg}$, a typical range in drug users. ${ }^{20}$ The same handling procedure was applied for hair samples taken from drug abusers.

\section{Extraction procedures}

About $20 \mathrm{mg}$ of drug-spiked hair or real hair was accurately weighted, put into 4-mL glass vials and spiked with 2-methylbenzylamine (IS), followed by the addition of $1 \mathrm{~mL}$ of $1 \mathrm{~mol} / \mathrm{L}$ $\mathrm{NaOH}$. The sealed vials were heated in a water bath at $70^{\circ} \mathrm{C}$ for 30 min to completely digest the hair and release the drugs. The digested hair solutions were transferred into 2-mL plastic centrifugal vials to remove any solid residuals, followed by the addition of $50 \mu \mathrm{L}$ of chloroform into each solution. We also added a small amount of solid $\mathrm{KCl}$ to the solutions until they became saturated in order to increase the recovery and to eliminate emulsification. The solution mixtures were vortexed for $1 \mathrm{~min}$, followed by centrifugation. The organic phases in the vials were drawn out directly with syringes for the analyses. concentration of $1 \mathrm{mg} / \mathrm{mL}$ (calculated in term of the amount of free base) in methanol, and further diluted with methanol to $100 \mu \mathrm{g} / \mathrm{mL}$ as stock solutions; heneicosane $\left(\mathrm{C}_{21} \mathrm{H}_{44}\right)$, an internal standard (IS) solution used in derivatization examinations, was dissolved in methanol at a concentration of $100 \mu \mathrm{g} / \mathrm{mL}$; 2-methyl-benzylamine (IS for hair analysis) was prepared at a concentration of $1 \mathrm{mg} / \mathrm{mL}$ in methanol as a stock solution. The derivatization was carried out in following steps: for standard samples, $5 \mathrm{ng}$ of amphetamines were dissolved in $20 \mu \mathrm{L}$ of chloroform in a 2-mL glass vial, followed by adding $10 \mu \mathrm{L}$ of MBTFA. The vial was then sealed with sealing film and placed in a microwave oven to heat up under $300 \mathrm{~W}$ for $3 \mathrm{~min}$. After derivatization, $1 \mu \mathrm{L}$ of the reacted solution was drawn for the analyses using GC/MS.

\section{Results and Discussion}

Optimization of the derivatization under microwave heating

We examined factors that might affect the derivatization efficiency, such as derivatizing solvents, amount of MBTFA, power and time of microwave heating. The area ratio of the derivatized products to IS $\left(\mathrm{C}_{21} \mathrm{H}_{44}\right)$, not reactive in the derivatization, was used as a parameter to calibrate the results. The derivatizations were tested in three different organic solvents, or a mixed solvent, they were chloroform, ethyl acetate and chloroform:pyridine with a 5:1 ratio. The derivatizations were the most efficient in chloroform:pyridine $(5: 1)$ or in chloroform. We chose chloroform as the derivatizing solvent.

The optimal ratio of MBTFA $(\mu \mathrm{L})$ to the analyte $(\mu \mathrm{g})$ was about one to one, set by trial-and-error experiments. The microwave power level and the duration of heating under the microwaves were set at $300 \mathrm{~W}$ for $3 \mathrm{~min}$ to achieve the maximum efficiency of derivatization without decomposing the products.

\section{Selection of extraction solvent}

The hair samples spiked with four amine drugs and IS were digested under an alkali condition. The extractions with organic solvents were conducted directly in the basic aqueous solutions with four organic solvents (they are toluene, cycle hexane, ethyl acetate, chloroform). Among them, there were no obvious differences in the extraction efficiency. We chose chloroform as the solvent for both extraction and derivatization, partially because of its greater density than water, allowing it to remain at the bottom of a tapered vial, which is very convenient for extraction, especially when handling with a very small volume. The ethyl acetate as a solvent is highly volatile, which makes quantification very difficult; $20,40,50,100$ and $200 \mu \mathrm{L}$ portions of chloroform containing $1 \mu \mathrm{g} / \mathrm{mL} \mathrm{C}_{21} \mathrm{H}_{44}$ as an IS were tested for extractions. The results showed that the smaller was the volume used in the extractions, the higher were the ratios of extracted drugs to IS. Therefore, the higher was the sensitivity of analysis achieved. Considering the ease of sampling from the extract, we chose $50 \mu \mathrm{L}$ for extraction. 


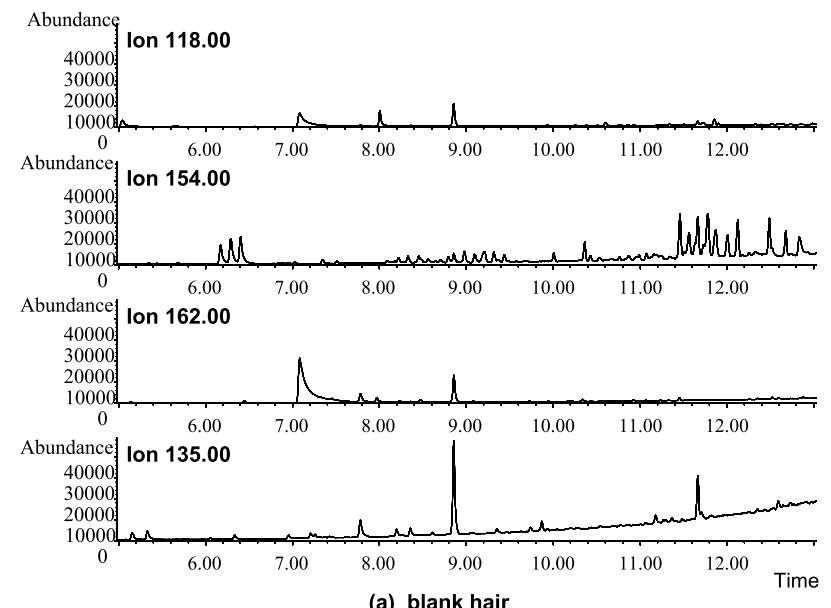

Table 2 Regression lines and detection limits for amphetamines spiked in hair

\begin{tabular}{lccccc}
\hline Drug & $\begin{array}{c}\text { Calibration curve } \\
y=a x+b\end{array}$ & $R^{2}$ & $\begin{array}{c}\text { Linear } \\
\text { dynamic } \\
\text { range/ } \\
\mathrm{ng} \mathrm{mg}^{-1}\end{array}$ & $\begin{array}{c}\text { LOD/ } \\
\mathrm{pg} \mathrm{mg}^{-1}\end{array}$ & $\begin{array}{c}\text { LOQ/ } \\
\mathrm{pg} \mathrm{mg}^{-1}\end{array}$ \\
\hline AM & $y=0.9106 x-0.0697$ & 0.9992 & $0.05-10$ & 20 & 50 \\
MAM & $y=1.3265 x-0.0322$ & 0.9996 & $0.05-10$ & 20 & 50 \\
MDA & $y=1.6543 x-0.0905$ & 0.9995 & $0.05-10$ & 20 & 50 \\
MDMA & $y=0.5075 x+0.0571$ & 0.9996 & $0.05-10$ & 20 & 50 \\
\hline
\end{tabular}

a. $y$, Peak-area ratio; $x$, concentration of drugs in hair $(\mathrm{ng} / \mathrm{mg})$.

Table 3 Relative recoveries and reproducibility of amphetamines at different concentrations

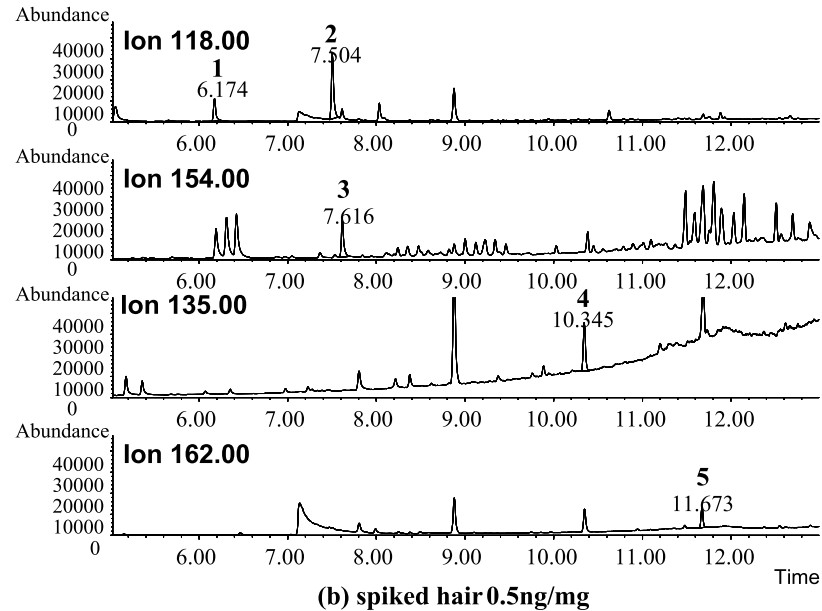

Fig. 1 Chromatograms of GC/MS-SIM of a blank (a) and spiked hair $(0.5 \mathrm{ng} / \mathrm{mg})$ (b) after MBTFA derivatization. 1, AM-TFA; 2 , IS-TFA; 3, MAM-TFA; 4, MDA-TFA; 5, MDMA-TFA.

Calibration curves using blank hair samples spiked with standards

We obtained calibration curves using the following procedures. First, a series of different amounts $(1,10,20,50,100$ and $200 \mathrm{ng}$ ) of the four amphetamine standards and $20 \mathrm{ng}$ of 2-methyl-benzylamine as an IS were spiked into the same amount $(20 \mathrm{mg})$ of washed blank hairs to prepare 6 calibrators. Second, the hair samples were digested in solutions containing $1 \mathrm{~mol} / \mathrm{L} \mathrm{NaOH}$. Third, the drugs from the dissolved hair solutions were extracted with $50 \mu \mathrm{L}$ of chloroform, and $20 \mu \mathrm{L}$ of the chloroform phases of extractions were transferred into new glass vials. Those samples were then derivatized with $10 \mu \mathrm{L}$ MBTFA under microwave heating. Lastly, the derivatized solutions were injected directly into a GC/MS, and the relative selected ion peak area (peak area of an analyte divided by that of the internal standard) vs. the concentration of the analyte in hair was plotted. The limits of detection (LODs) were determined based on the concentration in hair corresponding to a signal-to-noise ratio of 3:1. The limits of quantification (LOQ) were the lowest concentration over the linear range. Figure 1 shows the chromatograms and Table 2 lists the results.

\section{Reproducibility}

The daily reproducibility at different drug concentrations ( 5 replicates for each concentration) in hair was examined by spiking the same amount of drug standards and IS in five aliquots of blank hairs. The samples were processed with the

\begin{tabular}{llccc}
\hline \multirow{2}{*}{ Drug } & $\begin{array}{c}\text { Concentration/ } \\
\text { ng mg }^{-1} \text { in hair }\end{array}$ & $\begin{array}{c}\text { Relative } \\
\text { recovery, } \%\end{array}$ & $\begin{array}{c}\text { Intra-day } \\
\text { RSD, } \% \\
(n=5)\end{array}$ & $\begin{array}{c}\text { Inter-day } \\
\text { RSD, } \% \\
(n=6, d=3)\end{array}$ \\
\hline \multirow{2}{*}{ AM } & 5 & 96.5 & 2.6 & 6.6 \\
& 0.5 & 93.7 & 3.1 & 1.8 \\
MAM & 0.1 & 96.6 & 2.2 & 6.4 \\
& 5 & 95.8 & 7.2 & 4.1 \\
MDA & 0.5 & 103.2 & 4.9 & 7.5 \\
& 0.1 & 94.24 & 11.2 & 18.2 \\
& 5 & 102.4 & 1.8 & 5.1 \\
MDMA & 0.5 & 95.7 & 8.8 & 3.3 \\
& 0.1 & 102.7 & 13.5 & 6.9 \\
& 5 & 95.2 & 12.3 & 2.4 \\
& 0.5 & 97.1 & 10.0 & 3.7 \\
\hline
\end{tabular}

same procedures, and the peak-area ratios of the analytes to IS and the standard deviation of each analyte in hair were calculated. The inter-day reproducibility was examined within three days using 6 replicates of spiked hair samples at each concentration (Table 3). This procedure was repeated for different amounts of drugs to obtain the reproducibility at different drug concentrations. The relative standard variations $(\mathrm{CV} \%)$ for all drugs at almost all concentrations were less than $15 \%$ except that at a concentration of $0.1 \mathrm{ng} / \mathrm{mg}$ in hair for methamphetamine; the RSD\% was a slightly higher than the data (but less than $20 \%$ ). The data generally met the requirement for trace amount analysis.

\section{Relative recovery}

Relative recoveries at different drug concentrations in hair were calculated using the area ratio of an analyte to IS in spiked hair samples divided by the ratio obtained from the same amounts of the drug standards; the results are given in Table 3. The results show that the data are around $100 \%$, illustrating that the selection of IS was suitable for both the extraction and detection of the analytes. Loses in these processes were offset by the IS.

\section{Real hair analyses of amphetamines abuser}

Several hair samples from amphetamine drug abusers were collected and analyzed by the method presented in the paper. Methamphetamine was detected in most hair samples and its metabolite, amphetamine, was detected in some hair samples, depending on the amount of hair and the concentration of drugs in the hair. Amphetamine metabolized from methamphetamine was around $10 \%$, varying from the degree of the methamphetamine addiction. The higher was the percentage, the higher was the 
Table 4 Results of hair from abusers

\begin{tabular}{cccccccc}
\hline \multirow{2}{*}{$\begin{array}{c}\text { No. of } \\
\text { drug } \\
\text { abuser }\end{array}$} & $\begin{array}{c}\text { Hair } \\
\text { amount/ } \\
\text { mg }\end{array}$ & MAM & AM & $\begin{array}{c}\text { AM/ } \\
\text { MAM, } \\
\%\end{array}$ & MDMA concentrations in abuser's hair/ng mg & & MDA \\
\cline { 3 - 7 } & & & & $\begin{array}{c}\text { MDA/ } \\
\text { MDMA, } \\
\%\end{array}$ \\
\hline 1 & 20 & 12.26 & 0.77 & 6.28 & - & - & - \\
2 & 20 & 33.8 & 1.09 & 3.2 & - & - & - \\
3 & 1.5 & 2.1 & 0.22 & 10.5 & - & - & - \\
4 & 4.6 & 3.2 & 0.29 & 9.1 & - & - & - \\
5 & 7.7 & 0.24 & - & - & - & - & - \\
6 & 8.9 & 1.52 & - & - & - & - & - \\
7 & 22.2 & 1.11 & - & - & - & - & - \\
8 & 6.5 & 1.08 & - & - & - & - & - \\
9 & 21 & 0.45 & - & - & - & - & - \\
10 & 4.6 & 21.98 & 1.52 & 6.91 & 1.49 & 0.33 & 22.1 \\
\hline
\end{tabular}
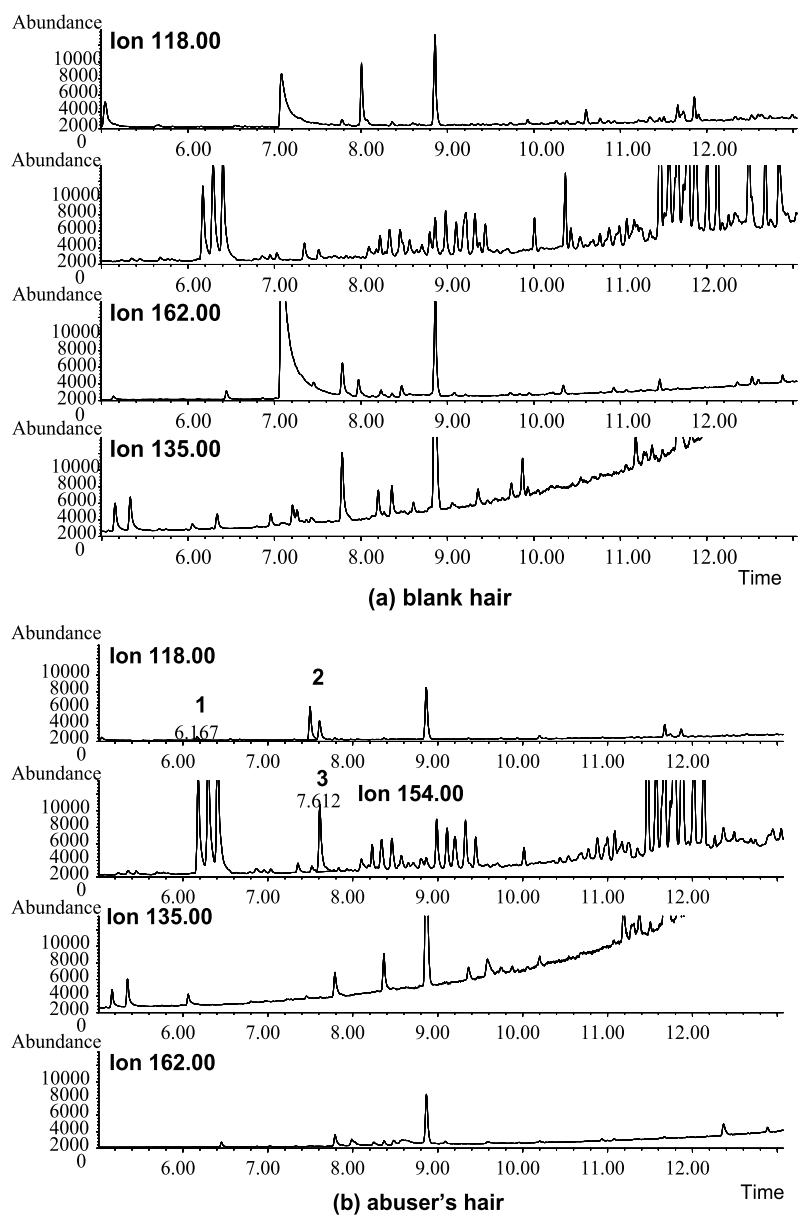

Fig. 2 Chromatograms of GC/MS-SIM of a blank (a) and abuser's hair (b) after derivatization. 1, AM-TFA; 2, IS-TFA; 3, MAM-TFA.

degree of the addiction. The smallest amount of collected hair was only $1.5 \mathrm{mg}$ (less than $10 \mathrm{~cm}$ in total length), in which the original drug of MAM, as well as its metabolite AM, were detected. The analysis results are listed in Table 4 . Figure 2 shows chromatograms of analysis for a real hair abuser (No. 3 in Table 4).

\section{Conclusions}

We report here on a simple, economic and sensitive procedure with good reproducibility for detecting amphetamines. Our procedure does not require laborious steps, such as $\mathrm{pH}$ adjusting, phase separation or solvent evaporation, used in most hair analysis procedures, which lead to sample losses and lower detection sensitivity. Hence, our procedure is more sensitive with a detection limit as low as $50 \mathrm{pg} / \mathrm{mg}$ in hair for the 4 amphetamines. In addition, our procedure is reliable, as demonstrated by its reproducibility with a relative standard deviation of less than $15 \%$. Moreover, our procedure is simpler and much less solvent and time consuming, and is more practical for detecting trace amounts of the amine drugs in the hairs of addicts.

\section{Acknowledgements}

We acknowledge support from China Scholarship Council and Cooperation-Item (No. 100410436) of Beijing Education Committee.

\section{References}

1. F. Tagliaro, Z. De Battisti, A. Groppi, Y. Nakahara, D. Scarcella, R. Valentini, and M. Marigo, J. Chromatogr., B, 1999, 723, 195.

2. Y. Nakahara, J. Chromatogr., B, 1999, 733, 161.

3. N. Shima, H. Kamata, M. Katagi, H. Tsuchihashi, T. Sakuma, and N. Nemoto, J. Chromatogr., B, 2007, 857, 123.

4. P. Meng, N. Fang, M. Wang, H. Liu, and D. D. Y. Chen, Electrophoresis, 2006, 27, 3210.

5. M. Nishida, A. Namera, M. Yashiki, and T. Kojima, $J$. Chromatogr., B, 2003, 789, 65.

6. M. Chèze, M. Deveaux, C. Martin, M. Lhermitte, and G. Pèpin, Forensic Sci. Int., 2007, 170, 100.

7. R. Gottardo, A. Fanigliulo, F. Bortolotti, G. D. Paoli, J. P. Pascali, and F. Tagliaro, J. Chromatogr., A, 2007, 1159, 190.

8. J. Y. Kim, K. S. Jung, M. K. Kim, J. I. Lee, and M. K. In, Rapid Commun. Mass Spectrom., 2007, 21, 1705.

9. S. Nakamura, M. Wada, B. L. Crabtree, P. M. Reeves, J. H. Montgomery, H. J. Byrd, S. Harada, N. Kuroda, and K. Nakashima, Anal. Bioanal. Chem., 2007, 387, 1983.

10. M. Nishida, M. Yashiki, A. Namera, and K. Kimura, $J$. Chromatogr., B, 2006, 842, 106.

11. F. Sporkert and F. Pragst, Forensic Sci. Int., 2000, 107, 129.

12. S. Gentili, M. Cornetta, and T. Macchia, J. Chromatogr., B, 2004, 801, 289.

13. S. Gentili, A. Torresi, R. Marsili, and M. C. T. Macchia, J. Chromatogr., B, 2002, 780, 183.

14. D. L. Allen and J. S. Oliver, Forensic Sci. Int., 2000, 107, 191.

15. M. Pujadas, S. Pichini, S. Poudevida, E. Menoyo, P. Zuccaro, M. Farrea, and R. D. L. Torre, J. Chromatogr., B, 2003, 798, 249.

16. M. Uhl, Forensic Sci. Int., 1997, 84, 281.

17. M. Uhl, Forensic Sci. Int., 2000, 107, 169.

18. R. Kronstrand, I. Nystrom, J. Strandbergb, and H. Druidb, Forensic Sci. Int., 2004, 145, 183.

19. N. Chen, Journal of Chinese People's Public Security University, 2005, 1,1 .

20. T. Cairns, V. Hill, M. Schaffer, and W. Thistle, Forensic Sci. Int., 2004, 145, 137. 